\title{
LA MEDIACIÓN VIRTUAL UN ESPACIO PROPICIO PARA LA FORMACIÓN EN INVESTIGACIÓN EN LA EDUCACIÓN SUPERIOR
}

\section{VIRTUAL MEDIATION A SPACE CONDUCIVE TO TRAINING IN RESEARCH IN HIGHER EDUCATION}

\section{A MEDIAÇÃO VIRTUAL É UM ESPAÇO FAVORÁVEL PARA O TREINAMENTO EM PESQUISA NO ENSINO SUPERIOR}

\section{César Augusto Aguirre León Corporación Universitaria Minuto de Dios UNIMINUTO caguirre@uniminuto.edu Bogotá D.C. - Colombia \\ Luis Juan Carlos García-Noguera Corporación Universitaria Minuto de Dios UNIMINUTO luis.garcian@uniminuto.edu Bogotá D.C. - Colombia \\ Zaily del Pilar García Gutiérrez Corporación Universitaria Minuto de Dios UNIMINUTO zaily.garcia@uniminuto.edu \\ Bogotá D.C. - Colombia \\ Erika Daniela Rodríguez Amórtegui Corporación Universitaria Minuto de Dios UNIMINUTO erika.rodriguez.a@uniminuto.edu Bogotá D.C. - Colombia}

Encuentre este artículo en:

http://revistas.uniminuto.edu/index.php/IYD

Para citar este artículo / To cite this article

Aguirre-León, C; García -Noguera, L; García -Gutiérrez, Z; Rodríguez- Amórtegui, E. (2020). La mediación virtual un espacio propicio para la formación en investigación en la educación superior. Inclusión \& Desarrollo, 7 (2), pp. 74-88
Fecha de recepción: 30 de enero de 2020 Fecha de aceptación: 13 de mayo de 2020 Fecha de publicación: 30 de junio de 2020 


\section{RESUMEN}

En el marco de la sociedad del conocimiento propiciada por el rápido desarrollo, masificación y apropiación de las tecnologías de la información y la comunicación, el fenómeno de la globalización y los avances que ha tenido la educación a distancia a partir de su incursión en la virtualidad, se presenta producto de una revisión documental, una reflexión crítica orientada a fundamentar el escenario de la mediación virtual como un espacio propicio para la formación en investigación en la educación superior. Para tal fin, se tiene en cuenta su origen y evolución, elementos que la caracterizan, desafíos a los que debe atender en el siglo $X X I$, entornos virtuales desde los que se puede desarrollar y presupuestos pedagógicos asociados a la formación en investigación que pueden ser considerados desde la mediación virtual. Este texto forma parte de los desarrollos alcanzados en el proyecto de investigación Impacto de los procesos de formación en investigación pedagógica en los estudiantes de último semestre de Licenciatura en Educación Infantil ofertados bajo la metodología "a distancia" y/o "virtual" en Bogotá D.C., aprobado en la VIII Convocatoria para el desarrollo y fortalecimiento de la investigación en la Corporación Universitaria Minuto de Dios. Ejecutado en la Vicerrectoría Bogotá Sur.

Palabras clave: mediación virtual, formación en investigación, educación superior, entornos virtuales, educación virtual.

\section{ABSTRACT}

Within the knowledge society framework fostered by the quick development, overcrowding and appropriation of information and communication technologies, the phenomenon of globalization and the progress that distance education has had since its incursion into virtuality, it is presented as a product of a documentary review, a critical reflection aimed at supporting the virtual mediation scenario as a favorable space for research training in higher education. For this purpose, its origin and evolution are taken into account, elements that characterize it, challenges to be addressed in the 21 st century, virtual environments where development can be done and pedagogical budgets associated with research training that can be developed from virtual mediation. This text is part of the developments achieved in the research project Impact of the training processes in pedagogical research on students in the last semester of Bachelor degree in Early Childhood Education offered under the "distance" and / or "virtual" methodology in Bogotá D.C, approved in the VIII Call for the development and strengthening of research in the Minuto de Dios University. This was carried out in a branch located in the south of Bogota

Keywords: virtual mediation, research training, higher education, virtual environments, virtual education.

\section{SUMÁRIO}

No marco da sociedade do conhecimento promovida pelo rápido desenvolvimento, massificação e apropriação das tecnologias da informação e comunicação, o fenômeno da globalização e os avanços que a educação a distância teve desde a sua incursão na virtualidade , é apresentado como produto de uma revisão documental, uma reflexão crítica que visa fundar o cenário da mediação virtual como um espaço adequado para o treinamento em pesquisa no ensino superior. Para tanto, são levados em consideração sua origem e evolução, elementos que a caracterizam, desafios a serem enfrentados no século XXI, ambientes virtuais a partir dos quais podem ser desenvolvidos e orçamentos pedagógicos associados ao treinamento em pesquisa que podem ser considerados da mediação virtual. Este texto faz parte dos desenvolvimentos 
alcançados no projeto de pesquisa Impacto dos processos de formação em pesquisa pedagógica no último semestre do Bacharelado em Educação Infantil oferecido sob a metodologia "à distância" e / ou "virtual" em Bogotá, DC , aprovado na VIII Chamada para o desenvolvimento e fortalecimento de pesquisas na Corporação Universitária Minuto de Dios. Executado na Vice-Reitoria Sul de Bogotá.

Palavras chave: mediação virtual, treinamento em pesquisa, ensino superior, ambientes virtuais, educação virtual. 


\section{Introducción}

El ser humano desde sus orígenes ha buscado transferir su conocimiento de generación en generación. Esta situación, lo ha llevado al desarrollo de sus capacidades e idear distintas maneras de conservar y difundir sus conocimientos, ideas y experiencias por diferentes medios, empleando distintas herramientas o mecanismos (José, 2016). Como señala Pérez (2014), a partir del desarrollo de la comunicación y la vida en comunidad, "los mitos fueron la primera tecnología humana para agrupar, sistematizar e informar de forma ordenada, coherente y cronológica los datos y acontecimientos humanos" (p. 71), lo que da una noción del ingenio de las personas para codificar y transmitir a los demás la información que posee, en busca de gestar conocimiento heredable a las futuras generaciones y salvaguardar su supervivencia.

En ese sentido, la necesidad continua de transmitir las experiencias acumuladas por la práctica, los nuevos descubrimientos y la evolución implícita en el desarrollo humano (Martin, 2007), motivó la creación de diferentes sistemas de comunicación, tales como: los jeroglíficos, la escritura cuneiforme, la imprenta, los diarios, la radio y el televisor, todos ellos con una significativa evolución a lo largo de su existencia, hasta llegar a las versiones conocidas en el siglo XXI (Olivar y Daza, 2007), ampliamente permeado por el avance vertiginoso de las Tecnologías de la Información y la Comunicación (TIC), a partir de los logros alcanzados en los siglos XIX y XX (Ávila, 2013).

Las TIC, como lo cita Cobo (2009) "se definen colectivamente como innovaciones en microelectrónica, computación, telecomunicaciones [...] que permiten el procesamiento y acumulación de enormes cantidades de información, además de una rápida distribución de la información a través de redes de comunicación" (p.305). Este concepto llevado a la realidad actual, se logra evidenciar en el rápido acceso y distribución de la información que ha posibilitado el internet y sus usos en herramientas tecnológicas tales como computadores, tablet o teléfonos inteligentes (Colás, 2003). Debido a esto, la etapa actual de la humanidad se le ha otorgado el nombre de sociedad de la información o sociedad del conocimiento (Alfonso, 2016).

De acuerdo con Forero (2009), la noción de sociedad de la información aparece por primera vez en el libro El advenimiento de la sociedad postindustrial, escrito por el sociólogo estadounidense Daniel Bell (1976), pero el término toma fuerza en 1995 cuando se incluye en la agenda de las reuniones del Grupo de los Siete (G7), y otros organismos de carácter internacional como la Organización para la Cooperación y el Desarrollo Económico (OCDE) y el Banco Mundial. Por otra parte, siguiendo a Burch (2005), el término sociedad del conocimiento ha sido adoptado por medios académicos, pues se considera que abarca una idea que va más allá del carácter económico.

De forma explícita la consideración de sociedad del conocimiento y sus alcances, se deja ver en las políticas institucionales desarrolladas por la Organización de las Naciones Unidas para la Educación, la Ciencia y la Cultura (UNESCO), en los Estándares de Competencia en TIC para Docentes, donde afirma que:

Para vivir, aprender y trabajar con éxito en una sociedad cada vez más compleja, rica en información y basada en el conocimiento, los estudiantes y los docentes deben utilizar la tecnología digital con eficacia [...] el docente es la persona que desempeña el papel más importante en la tarea de ayudar a los estudiantes a adquirir esas capacidades. Además, es el responsable de diseñar tanto oportunidades de aprendizaje como el entorno propicio en el aula que faciliten el uso de las TIC (UNESCO, 2008, p.2).

Tal mirada deja ver que, la educación en sus distintas categorías (formal, educación para el trabajo y desarrollo humano, e informal) y niveles 
de la cadena de formación (preescolar, básica, media y superior), ha sido uno de los escenarios donde más impacto ha tenido el avance de las TIC (Hernández, 2017), lo que ha motivado en el marco de la globalización, el continuo avance y resignificación de las modalidades de enseñanza a partir de nuevas formas de entender y asumir el proceso de aprendizaje (García, 2012), de un contexto ampliamente marcado por el predominio de la educación presencial a uno más inclusivo y de mayor acceso desarrollado desde la educación virtual (Dueñas, 2017; Cardona, 2002), vista desde expresiones tales como la educación e-learning o la educación b-learning, entre otras.

En esa dinámica, el desarrollo de propuestas educativas virtuales de forma total o parcial, tiene en la mediación virtual uno de sus principales pilares para la generación de conocimiento y el cumplimento de las funciones misionales de la educación superior: docencia, investigación y proyección social. Esta situación, obliga a la reflexión sobre los procesos de construcción de conocimientos y desarrollo de competencias a través de la mediación virtual, en especial los relacionados con la formación en investigación, al ser vista esta como un elemento básico para el desarrollo de los procesos de enseñanza asociados al ejercicio de la docencia en los programas académicos (pregrado y posgrado) y el cumplimiento de la responsabilidad social universitaria (Ayala, 2011).

\section{Medición virtual}

La mediación del aprendizaje, puede ser entendida como una apuesta pedagógica de tipo constructivista que enmarca el rol del docente como propiciador de situaciones de aprendizaje de las cuales el estudiante progresivamente va dando cuenta, lo que propicia la formación de un aprendiente autónomo (Tunnermann, 2011). Este tipo de proceso, de acuerdo con Espinosa (2016), particulariza el proceso de aprendizaje, al trazar un curso de acción entre lo que debe ser aprendido y las competencias que posee el alumno.
La mediación virtual, retoma los elementos característicos de esta acción pedagógica y la enmarca en el contexto de la educación virtual. En tal sentido, de acuerdo con Luque (2009), corresponde a uno de los principales dispositivos usados en los distintos entornos de aprendizaje virtual (EVA) a partir del avance de las TIC, para poder desarrollar de manera interactiva los procesos formativos diseñados en las aulas virtuales para la construcción y desarrollo de las competencias requeridas.

A partir de los desarrollos que han tenido las TIC en el siglo XXI, las mediaciones virtuales continuamente se han tenido que revisar $y$ rediseñar de acuerdo con las nuevas apuestas tecnológicas y sus potencialidades formativas (Ramírez y Chávez, 2012). Esta situación, ha llevado a la construcción de recursos virtuales más dinámicos, de fácil acceso, riqueza en su contenido y múltiples espacios para la interacción académica entre los distintos actores que convergen en el proceso de aprendizaje, de acuerdo con las particularidades del aula en correspondencia con las apuestas institucionales (Rodríguez-Hoyos y Álvarez, 2013).

\section{Entornos de aprendizaje con mediación virtual}

El desarrollo de las TIC en el siglo XX y XXI ha incidido de manera directa en el desarrollo de EVA construidos desde la virtualidad (Pérez y Telleria, 2012). Este elemento fundante, ha dado forma a diferentes herramientas, estrategias y desarrollos informáticos que han enriquecido, diversificado y hecho más atractivas las propuestas educativas propias de la educación a distancia y de la educación presencial con mayor o menos uso de recursos virtuales.

\section{E-Learning}

El E- Learning, abreviación de Electronic Learning, traducido como aprendizaje electrónico o educación en línea, es un entorno de aprendizaje virtual que tiene sus orígenes en el auge tecnológico 
que hubo en la década de los noventa del siglo XX y su aplicación en el campo de la educación (Aiello y Willem, 2004), donde empieza a tomar forma la enseñanza mediante cursos contenidos en CD-ROM, casetes y disquetes. En el siglo XXI con el desarrollo de internet, se vislumbraron los primeros conceptos y desarrollos E - Learning de aulas y campus virtuales (Bustos y Coll, 2010).

De acuerdo a García-Peñalvo y Pardo (2015), el E- Learning está desarrollado desde tres características fundamentales:

1. Trabaja en red, por lo tanto, se actualiza constantemente, es de acceso inmediato y almacena gran cantidad de información.

2. El usuario final recibe la información a través del uso de ordenadores o tecnología que tenga acceso a internet

3. Tiene una visión más amplia que el aprendizaje tradicional, pues permite la continua asimilación del conocimiento. De igual manera, su evolución tecnológica ha permitido crear ecosistemas tecnológicos donde es posible interactuar con pares a distancia y en tiempo real.

En su proceso de avance e innovación, es posible distinguir varias generaciones del E- Learning, en donde las herramientas usadas para su implementación constituyen el punto de quiebre que determina su evolución. En ese sentido, García- Peñalvo y Pardo (2015), destacan las siguientes generaciones, como las más importantes de acuerdo con su impacto en la comunidad y la manera en que se fueron aplicando en atención a las necesidades del momento.
Tabla1

Generaciones del E- Learning

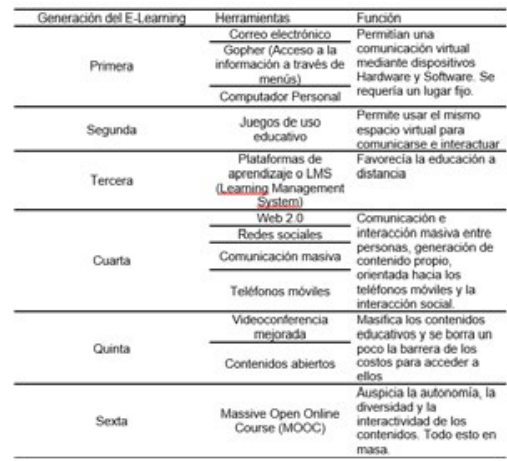

Fuente: elaboración propia con datos de GarcíaPeñalvo y Pardo (2015)

Las consideraciones sobre el E- Learning permiten ver que la tecnología en la medida en que se ha ido desarrollando, ha influido en la masificación del tráfico de contenidos (RuedaLópez, 2007), creación de variada oferta de conocimiento online de manera paga o gratuita, y además ha involucrado a cada sujeto como actor principal de su proceso de formación. Esta última manifestación ampliamente ilustrada en los desarrollos que ha tenido la educación a distancia (Rivera-Vargas, Alonso-Cano y Sancho-Gil, 2017).

\section{M-Learning}

El Mobile Learning o M-Learning, surge como respuesta al auge tecnológico del siglo XXI a partir de la evolución del E - Learning, en donde la tendencia a crear dispositivos móviles multifuncionales, reemplaza, fusiona o comprime poco a poco los aparatos que existían a finales del siglo pasado y las funciones específicas que los caracterizaban (Besantes, Naranjo, Gallegos, Betancourt y Quiña, 2017). Esta modalidad de aprendizaje virtual, traducida como aprendizaje electrónico móvil, se apoya en el uso de dispositivos móviles conectados a redes inalámbricas que facilitan la transmisión de información, acceso, interacción, consulta y colaboración en los contenidos de manera inmediata desde cualquier lugar (Pina et al., 2016). Ejemplo de los dispositivos 
con las características esenciales del M-Learning, son los teléfonos inteligentes, las tablets y laptops, ya que reúnen los atributos necesarios de portabilidad y conexión remota para la interacción oportuna.

Otros atributos del M-Learning según Pina et al. (2016) es que, bajo esta modalidad se puede impartir un conocimiento de manera personalizada, respetando el espacio y tiempo del alumno, puesto que puede acceder a sus materiales desde cualquier lugar en el momento que mejor le convenga, además se ajusta al estilo de aprendizaje de cada persona debido a la facilidad y flexibilidad de acceso disponible.

De igual manera, algunas barreras a las que se expone el M-Learning, son: falta de conexión, miedo de los usuarios a la pérdida o robo de sus dispositivos, alto costo de los dispositivos más eficientes y temor por parte de los usuarios a que el aprendizaje no sea efectivo.

En el contexto universitario de acuerdo con Oliva, Narváez y Buhring (2016), el uso de dispositivos móviles "genera una instancia de interacción permanente entre el sujeto involucrado en el ambiente educativo con la información mediada por la tecnología que se transforma en unidades ergonómicas y funcionalmente multifacéticas" (p. 374). Lo que ayuda a la comunicación en tiempo real entre docentes y estudiantes, bien sea de manera personalizada o grupal.

\section{B-Learning}

Tradicionalmente las modalidades de educación a virtual y presencial, han sido opuestas por la manera como llegan a los estudiantes. El Blended Learning o B- Learning, traducido como aprendizaje semipresencial, es una apuesta formativa mixta tendiente a la construcción de un entorno de aprendizaje que retoma $y$ fusiona elementos de la educación presencial y la educación online (Salinas, De Benito, Pérez y Gisbert, 2018), de acuerdo a los procesos formativos que deben desarrollarse en los cursos académicos matriculados por los estudiantes.

Cuando en el B-Learning se fusionan las modalidades virtual y presencial, el docente, quien ejerce el rol de tutor en el proceso de construcción del aprendizaje (Cano, 2009), es el responsable de diseñar, acompañar y retroalimentar el proceso de aprendizaje que vivirá el estudiante con actividades virtuales y presenciales (Martí, 2009). De igual manera, el alumno debe tener claro sus objetivos de aprendizaje, tareas a desarrollar y administración de los tiempos (Bartolomé, 2004). Así, el B-Learning se puede definir como la combinación entre el sistema educativo presencial (en donde el docente se encarga de orientar una tutoría) y el sistema a distancia con mediación virtual (en donde el estudiante asume la responsabilidad de desarrollar los contenidos de su curso).

Graham (citado por Salinas, 2012) diferencia 4 tipologías de B-Learning, de acuerdo al nivel de implementación o campo de acción.

Tabla 2

Tipologías de B-Learning

\begin{tabular}{ll}
\hline Nivel & Característica \\
\hline A nivel actividad & $\begin{array}{l}\text { Actividades mediadas entre elementos de } \\
\text { comunicación por ordenador y factores de } \\
\text { presencialidad }\end{array}$ \\
\hline A nivel de curso & $\begin{array}{l}\text { Un curso B-Learning combina tutorias presenciales } \\
\text { con actividades en plataformas virtuales }\end{array}$ \\
\hline $\begin{array}{l}\text { A nivcl de programa } \\
\text { formativo }\end{array}$ & $\begin{array}{l}\text { Un programa B-Locarning pucdc ofrccer un } \\
\text { porcentaje de formación de manera virtual (la mayor } \\
\text { parte) y otro porcentaje de manera presencial. Este } \\
\text { puede intercalar actividades virtuales con } \\
\text { presenciales u ofrecer la parte virtual al inicio y dejar } \\
\text { lus encuentros presenciales para el final (o } \\
\text { viceversa) }\end{array}$ \\
\hline A nivel institucional & $\begin{array}{l}\text { Ofrecer un modelo B-Learnina en una institución } \\
\text { aplica cuando se reestructura el número de horas } \\
\text { presenciales y se ofrece un itinerario en línea. }\end{array}$ \\
\hline
\end{tabular}

Fuente: elaboración propia con datos de Salinas (2012)

Finalmente, los recursos diseñados y utilizados en entornos B-Learning tales como, Objetos Virtuales de Aprendizaje (OVA) o herramientas interactivas, pueden desarrollar en los estudiantes habilidades significativas en su formación personal y profesional, tales como: 
trabajo en equipo, comunicación asertiva y resolución de problemas.

\section{U-Learning}

El U-Learning, Ubiquitous Learning, traducido como aprendizaje ubicuo, hace referencia a una forma de aprendizaje continuo producto de las interacciones establecidas en un aula virtual desde distintos dispositivos tecnológicos (Flores y García, 2017), entre los cuales se puede encontrar: móviles, computadores o tablet. Cabe resaltar que, no se enmarca en la capacidad de estar en diversos lugares al tiempo, hace alusión a la posibilidad de dar continuidad al proceso formativo, en cualquier momento, lugar o situación (Hidalgo, Orozco y Daza, 2015).

Estatendenciadeaprendizaje, sedacomoproducto de la evolución de las TIC, en particular por la expansión de la conectividad y la masificación de los recursos tecnológicos que permiten el acceso a distintos tipos de entono virtual de aprendizaje. En esa dinámica, se diferencia del E-Learning y MLearning porque su espectro es mucho más amplio, lo que hace posible dimensionar el aprendizaje más allá del uso de los teléfonos móviles (Báez y Clunei, 2019).

Si bien el U-Learning, guarda correspondencia con otros EVA, es también posible señalar algunas características que le son inherentes:

Tabla 3

Características aprendizaje Ubicuo

\begin{tabular}{ll}
\hline Característica & Descripción \\
\hline Permanencia & $\begin{array}{l}\text { De forma permanente se puede contar con los } \\
\text { materiales, actividades e información consignada } \\
\text { en el proceso de aprendizaje diseñado desde la } \\
\text { mediación virtual }\end{array}$ \\
\hline Accesibilidad & $\begin{array}{l}\text { Se puede acceder de manera continua a través de } \\
\text { distintos dispositivos tecnológicos, más allá de las } \\
\text { condiciones de lugar o momento }\end{array}$ \\
\hline Inmediatez & $\begin{array}{l}\text { Al buscar la información, materiales o actividades, } \\
\text { estas bajo condiciones óptimas de conectividad y } \\
\text { calidad en las herramientas tecnológicas de uso, } \\
\text { estarán de forma continua e inmediata al alcance de } \\
\text { quien la necesite }\end{array}$ \\
\hline Interactividad & $\begin{array}{l}\text { El conocimiento es construido desde la interacción } \\
\text { establecida entre los distintos actores educativos a } \\
\text { partir de las actividades planeadas por el mediador }\end{array}$ \\
\hline Adaptabilidad & $\begin{array}{l}\text { Este entorno se adapta a las particularidades } \\
\text { formativas de los estudiantes acorde con el } \\
\text { desarrollo previsto de contenidos, habilidades y } \\
\text { destrezas para dar forma a las competencias }\end{array}$ \\
\hline
\end{tabular}

Fuente: elaboración con datos de Yahya, Ahmad y Abb (2010)

\section{Experiencias de mediación virtual}

La mediación virtual en la formación de profesionales, constituye un espacio fundamental en la generación de conocimiento. Según MasTorelló y Olmos-Rueda (2016), el uso de las TIC ha permitido incursionar en nuevas maneras de transmitir instrucciones, permitiendo generar nuevas dimensiones en donde se rompe el paradigma del tiempo - espacio. En esa perspectiva, hoy en día las redes permiten impartir los saberes sin necesidad de recurrir siempre a la presencialidad y faculta la organización de tiempos de acuerdo a las necesidades del estudiante, o en el caso de la educación tradicional se puede apoyar de la virtualidad para tener comunicación más eficiente y rápida con los estudiantes.

Entrelas diversas experiencias demediación virtual que es posible hallar en bases de datos y repositorios digitales, son notorios los alcances de las experiencias que se detallan a continuación por los logros obtenidos en el proceso de aprendizaje de los estudiantes:

1. Prácticas innovadoras de enseñanza con mediación TIC que generan ambientes creativos de aprendizaje (Lozano, 2014) : a partir de la ejecución del proyecto de investigación Ambientes creativos de aprendizaje con mediación de TIC, para la enseñanza innovadora de Lengua Castellana, Matemáticas y Ciencias Naturales, derivados de la sistematización de proyectos de aulas, desarrollado entre el año 2012 y 2014, por parte del grupo de investigación Cibereducación de la Católica del Norte Fundación Universitaria - Colombia, teniendo en cuenta experiencias significativas gestadas por maestros de los departamentos de Antioquia y Chocó, se evidenció que el uso de la mediación virtual mejora en los estudiantes sus procesos de concentración y compromisoacadémico, desarrollasuscapacidades creativas y provoca cambios que los aproximan a las características de la sociedad del conocimiento. 
2. Políticas universitarias, Gestión y Formación Docente en Educación a Distancia. Hacia una Pedagogía de la virtualización (Copertari, Sgreccia y Segura, 2011): realizan un análisis de la transición que está sufriendo la universidad (específicamente la Universidad Nacional de Rosario - Argentina) en el estilo de enseñanza para docentes en ejercicio y en formación. Lo que ha representado un desafío, al tener que reinventarse para acoplar su oferta a la demanda del mercado educativo. En esa dinámica, se logra concluir que, el apoyo de la virtualidad en el proceso de formación está reconfigurando los estilos de aprendizaje y enseñanza. Visto de esa manera, el camino a la virtualidad está claro y prácticamente es un hecho su imperiosa aplicabilidad, debido a los avances tecnológicos que constantemente se filtran en el quehacer cotidiano, con el objeto de contribuir al acceso fácil y rápido a la información, por lo tanto, es una herramienta que debe ser aprovechada para la educación.

3. Propuesta de buenas prácticas de educación virtual en el contexto universitario (Ruiz y Antonio, 2016): este trabajo realizado en Venezuela, deja ver un ejercicio de revisión y reflexión a partir de la recopilación y revisión de 18 prácticas docentes orientadas a la formación virtual en educación superior y presenta una propuesta de buenas prácticas para los procesos formativos desarrollados a través de aulas virtuales. Concluye que las prácticas desarrolladas desde entornos E-Learning son de gran valor y calidad para el desarrollo de los procesos formativos propios de la educación superior.

4. Entornos virtuales de aprendizaje como apoyo a la enseñanza presencial para potenciar el proceso educativo (Rodríguez y Barragán, 2017): el estudio desarrollado en la Universidad Católica de Cuenca - Ecuador, buscó promover el uso de entornos virtuales en los procesos de enseñanza aprendizaje (EVE/A) dentrodelaformación deaprendientesautónomos en el programa de Ingeniería Empresarial. A partir del diseño y ejecución de entornos virtuales, y su posterior evaluación, se pudo apreciar mayor interés de los estudiantes de forma individual o grupal en las actividades virtuales propuestas, su actitud frente a los retos educativos propuestos y mejoras en su desempeño.

5. La perspectiva de estudiantes en línea sobre los entornos virtuales de aprendizaje en la educación superior (Blanco y Anta, 2016): la investigaciónejecutadaenlaUniversidadAutónoma de Barcelona, a partir de la exploración cualitativa de la percepción que tienen los estudiantes de posgrado sobre los beneficios y dificultades de los EVA, concluye que estos espacios de formación son considerados como una posibilidad de aprendizaje enmarcada en principios de flexibilidad, eficiencia, adecuación y rigurosidad.

En conjunto, al valorar las diferentes experiencias, es posible afirmar que, se asiste actualmente a una época en donde se puede generar y transmitir conocimiento incitando a la autonomía de los estudiantes gracias al avance de las TIC, la expansión de la conectividad al internet y las redes de información que actualmente convocan a variados EVA, lo que a su vez repercute en los desarrollos de la educación superior a distancia, con propuestas de calidad que llevan a los distintos actores a ser partícipes de la sociedad del conocimiento.

\section{Desafíos de la medición virtual}

Es evidente que la educación actual tiene un reto (y compromiso) bastante grande respecto a la reinvención de los contenidos, gracias a la necesidad de suplir la demanda de información y conocimiento que tiene la comunidad educativa del siglo XXI, pues día tras día se debe pensar en crear contenidos dentro de los currículos universitarios, que sean funcionales y que empleen la mediación virtual para la optimización de resultados. En esa dinámica Fainholc (2006), menciona tres desafíos importantes para la implementación de la mediación virtual en educación: El diseño del curso 
y las situaciones de enseñanza y aprendizaje, Remover mentalidades, y Conocer las habilidades y competencias.

\section{El diseño del curso y las situaciones de enseñanza}

Este es uno de los compromisos fundamentales en el desarrollo de la mediación virtual. El diseño de situaciones de enseñanza que recuperen las competencias de los estudiantes y potencien su desarrollo, constituye un elemento clave en el proceso de aprendizaje (García, 2011). En tal sentido, la mediación debe potenciar a partir de herramientas tecnológicas interactiva la cercanía del estudiante al conocimiento, la recuperación de los saberes previos, la conceptualización, comprensión, transferencia y evaluación de lo aprendido (Villareal-Treviño, 2006).

\section{Remover mentalidades}

Busca el cambio de mentalidad en los actores de la educación (docentes - tutores y estudiantes), respecto a la introducción de herramientas virtuales, para sacar el máximo provecho del conjunto de posibilidades que estas ofrecen dentro de sus actividades cotidianas. Este desafío, está articulado a una de las situaciones de analfabetismos del siglo XXI, el analfabetismo informático que muchas veces tiende a limitar por falta de conocimiento o creencias asociadas al uso de ambientes virtuales en procesos de aprendizaje (Crovi, 2002). La superación de las creencias, se puede constituir en un impulso para dar forma a proceso de aprendizaje que aprovechen la riqueza de la virtualidad.

\section{Conocer las habilidades y competencias}

Conocer las habilidades y competencias, debe ser considerado como uno de los ejercicios metacognitivos más valiosos para poder direccionar las acciones de aprendizaje. Solo desde este punto de partida, es posible diseñar cursos de acción que de manera asertiva construyan aprendizajes profundos y trascendentes (Fasce, 2007). De lo contrario, los conocimientos solo darán forma a un aprendizaje superficial, más de corte memorístico que significativo.

La formación mediada por la virtualidad es una de las modalidades de educación superior más fuerte en los actuales momentos. Pero su implementación, requiere una continua revisión y alistamiento de los procesos que en ella se desarrollan, para ir acorde con el momento histórico, los intereses de los estudiantes y las expectativas formativas de la sociedad.

\section{Presupuestos pedagógicos para la mediación virtual de formación en investigación}

La mediación virtual en la educación superior, es en el siglo XXI uno de los principales y más pertinente recurso pedagógico para hacer posible las apuestas curriculares (Muñoz, 2016). Esta consideración se puede fundamentar a partir del reconocimiento de las características de la sociedad del conocimiento a la cual se asiste actualmente y a su vez los intereses tecnológicos propios de una generación marcada por un entorno ampliamente permeado por las TIC, lo que hace que sea de mayor interés los procesos educativos que de forma parcial o total incorporan el uso de entornos virtuales.

De igual manera, en el marco de la política de créditos académicos en la educación superior, el desarrollo de procesos de mediación a través de aulas virtuales, puede ser considerado en los programas ofertados en la modalidad presencial como un recurso útil para el aprovechamiento de las horas de aprendizaje autónomo (Sierra, 2012). Con más razón aún en los programas a distancia donde las horas de aprendizaje autónomo es mayor, llegando incluso en algunos casos a ocupar la totalidad de horas del crédito académico.

Enesemismosentido,altenerpresentecomo señala Rojas y Aguirre (2015) que la investigación constituye una de las funciones misionales de las 
universidades y que en los actuales momentos su apropiación y desarrollo es vital para el desarrollo de los individuos en su entorno profesional, es posible considerar la mediación virtual como un recurso para desarrollar procesos de formación en investigación (Aguirre, 2016). Esta consideración se puede llegar a dimensionar desde dispositivos que conduzcan a la formación de las competencias y el desarrollo de procesos de investigación formativa que optimicen el desempeño esperado.

Para el desarrollo de los procesos de mediación de la formación en investigación a partir del diseño de un aula virtual indistintamente del EVA al cual se tenga acceso, es conveniente de acuerdo con Londoño (2011), Delgado y Solano (2009) y Del Pradoy Doria (2015), tener en cuenta consideraciones pedagógicas con relación a: modelo pedagógico, diseño de aulas, compromiso de los docentes y espacios de interacción.

Tabla 4

Consideraciones pedagógicas para la mediación virtual de la formación en investigación

\begin{tabular}{ll}
\hline Consideración & Aspectos a tener en cuenta \\
\hline Modelo pedagógico & La definición del modelo pedagógico asumido \\
& desde la perspectiva institucional, debe dejar \\
& definido los elementos pedagógicos, didácticos y \\
curriculares a tener en cuenta para en el marco de & la virtualidad (Castro, Gatebiel, Gutiérrez y \\
& Hernández, 2005), dar forma al ideal de formación, \\
& selección de contenidos, metodologías de \\
& enseñanza, secuenciación de los contenidos, \\
& relación de los actores educativos y evaluación del \\
proceso de aprendizaje. & De igual manera, es convente considerar las pautas \\
& a tener en cuenta para potenciar el desarrollo de \\
& EVA. \\
\hline A partir de los recursos tecnológicos institucionales, \\
es conveniente considerar el diseño de aulas \\
virtuales colaborativas, flexibles y dinámicas. \\
Desde esa perspectiva, el diseño de las aulas debe \\
responder al desarrollo actual de la tecnología y a \\
espacios de formación e interacción académica \\
adecuados (Castro, Guzmán y Casado, 2007). \\
Dentro de su desarrollo es posible sugerir espacios \\
de interacción tales como foros, wiki y chat. El uso \\
de recursos de evaluación tales como portafolios, \\
estudio de caso y pruebas virtuales, y el acceso a \\
recursos de contenido entre los que se pueden \\
hallar libros digitales, bases de datos y repositorios \\
académicos virtuales. \\
Más allá del diseño y recursos del aula se debe \\
propugnar por propuestas que estimulen la \\
motivación intrínseca de los estudiantes (Elizondo, \\
Rodríguez y Rodríguez, 2018), en pro de asumir las \\
actividades como retos de aprendizaje que \\
estimulen al desarrollo de las comoetencias. \\
La labor de los docentes - tutor en el escenario de \\
la formación a través de EVA, es un elemento de \\
gran importancia para asegurar la calidad del \\
recurso virtual (Silva, 2010). \\
\hline docentes & \\
\hline & \\
&
\end{tabular}

\begin{tabular}{|c|c|}
\hline & $\begin{array}{l}\text { Visto de esa manera, el compromiso de los } \\
\text { docentes, puede dar forma significativa a la apuesta } \\
\text { del diseño del aula virtual, su contenido, } \\
\text { administración y retroalimentación. }\end{array}$ \\
\hline $\begin{array}{l}\text { Compromiso de los } \\
\text { estudiantes }\end{array}$ & $\begin{array}{l}\text { El compromiso académico de los estudiantes es tal } \\
\text { vez, el de mayor envergadura al tener en cuenta } \\
\text { que en el escenario de la virtualidad son quienes } \\
\text { van a manejar su proceso de aprendizaje más allá } \\
\text { de las condiciones de tiempo, modo y lugar } \\
\text { (Donolo. Chiecher.v Rinaudo 2004). }\end{array}$ \\
\hline Espacios de interacción & $\begin{array}{l}\text { Los espacios del aula para la interacción académica } \\
\text { entre los distintos autores, es un elemento vital para } \\
\text { la construcción de aprendizajes, validación de } \\
\text { experiencias y construcción de proyectos. En tal } \\
\text { sentido el aula virtual debe disponer de múltiples } \\
\text { espacios de interacción de los estudiantes con sus } \\
\text { iguales y con el docente (Colina, 2008), para de } \\
\text { forma ágil, dinámica y de fácil acceso posibilitar } \\
\text { desde el aula el encuentro académico y el } \\
\text { desarrollo de destrezas (Delgado y Solano, 2009). }\end{array}$ \\
\hline
\end{tabular}

El tener en cuenta que todo proceso educativo está implícita o explícitamente permeado por un proceso pedagógico que lo hace posible, permite dimensionar la importancia que tiene la consideración de los supuestos pedagógicos que pueden acompañar la mediación virtual de la formación en investigación. De igual manera, son aspectos de continua revisión y validación a partir del surgimiento de nuevas apuestas metodológicas virtuales, losinteresesformativos de losestudiantes y las apuestas de las instituciones con relación al cumplimiento de sus funciones misionales.

\section{Conclusiones}

La formación a través de la medición virtual, es sin lugar a dudas en el siglo XXI una oportunidad para acceder a la sociedad del conocimiento, acrecentar y validar aprendizajes, y desarrollar nuevas experiencias de interacción en red que potencien la enseñanza recíproca.

Considerar la posibilidad de formar en investigación desde la mediación virtual, es reconocer que, este tipo de recurso es apto no solo para el desarrollo de la docencia, también hace posible el desarrollo de experiencias de investigaciónformativa quevalidanlos aprendizajes en metodología y técnicas de la investigación científica, y enriquecen las competencias en investigación desde experiencias adelantadas a partir de estudios de caso, estados de arte o proyectos de investigación adelantados desde aulas virtuales con determinadas características de EVA. 
Dar cabida a la formación en investigación de manera teórica y práctica desde la virtualidad es propiciar el reconocimiento de los EVA como escenarios académicos que pueden alcanzar la calidad de los procesos formativos adelantados tradicionalmente desde la presencialidad y que, pueden aportar elementos para cualificar conocimientos, destrezas y valores en distintas dimensiones del ser humano. 


\section{Referencias bibliográficas}

Aiello, M., y Willem, C. (2004). El blended learning como práctica transformadora. Pixel-Bit. Revista de Medios y Educación, (23), 21-26.

Alfonso, I. (2016). La Sociedad de la Información, Sociedad del Conocimiento y Sociedad del Aprendizaje. Referentes en torno a su formación. Bibliotecas. Anales de investigación, 12(2), 235-243.

Aguirre, C. (2017). Desarrollo de competencias de investigación en estudiantes de educación superior con la mediación de herramientas de m-Learning \& e-Learning. Inclusión \& Desarrollo, 4(1), 68-83.

Ayala, M. (2011). Responsabilidad social universitaria. Realidad y Reflexión, 2011, Año. 11, nùm. 33, p. 61-72.

Ávila, W. (2013). Hacia una reflexión histórica de las TIC. Hallazgos, 10(19), 213-233.

Báez, C. y Clunei, C. (2019). Una mirada a la Educación Ubicua. RIED. Revista Iberoamericana de Educación a Distancia, 22(1). Bartolomé, A. (2004). Blended Learning. Conceptos básicos. Pixel-Bit, Revista de Medios y Educación, 23, 7-20. https://idus. us.es/xmlui/handle/11441/55455

Bell, D. (1976). El advenimiento de la sociedad post-industrial: un intento de prognosis social. Madrid, España: Alianza editorial. Besantes, A., Naranjo, M., Gallegos, M., Betancourt, S. y Quiña, J. (2017). Aprendizaje móvil en el aula. Ecos de la academia, 3(05), 60-69.

Blanco, A. y Anta, P. (2016). La perspectiva de estudiantes sobre los entornos virtuales de aprendizaje en la educación superior. Innoeduca: international journal of technology and educational innovation, vol. 2, no 2, p. 109-116.

Burch, S. (2005). Sociedad de la información/Sociedad del conocimiento. Palabras en juego, 54-78. https://analfatecnicos.net/ archivos/76.SociedadDeLalnformacionYConocimiento-SallyBurch.pdf

Bustos, A. y Coll, C. (2010). Los entornos virtuales como espacios de enseñanza y aprendizaje. Una perspectiva psicoeducativa para su caracterización y análisis. Revista mexicana de investigación educativa, 15(44), 163-184.

Cano, R. (2009). Tutoría universitaria y aprendizaje por competencias: ¿Cómo lograrlo? Revista electrónica interuniversitaria de formación del profesorado,12-1 (28), 181-204.

Cardona, G. (2002). Tendencias educativas para el siglo XXI. Educación virtual, online y@ learning. Elementos para la discusión. Edutec. Revista electrónica de tecnología educativa, (15), a025-a025.

Castro, G., Catebiel, V., Gutiérrez, E. y Hernández, U. (2005). La Red de Investigación Educativa: hacia una construcción curricular alternativa en procesos de formación avanzada. Revista ieRed, I, 3, 1-16.

Castro, S., Guzmán, B. y Casado, D. (2007). Las TIC en los procesos de enseñanza y aprendizaje. Laurus, 13(23), 213-234.

Cobo, J. (2009). El concepto de tecnologías de la información. Benchmarking sobre las definiciones de las TIC en la sociedad del conocimiento. Zer: Revista de estudios de comunicación, 14(27). https://www.ehu.eus/ojs/index.php/Zer/article/ view/2636/2182

Colás, M. (2003). Internet y aprendizaje en la sociedad del conocimiento. Comunicar: Revista científica iberoamericana de comunicación y educación, (20), 31-35.

Colina, L. (2008). Las TIC en los procesos de enseñanza-aprendizaje en la educación a distancia. Laurus, 14(28), 295-314.

Copertari, S., Sgreccia, N. y Segura, M. L. (2011). Políticas universitarias, Gestión y Formación Docente en Educación a Distancia. Hacia una Pedagogía de la virtualización. Revista de Educación a Distancia, (27). https://revistas.um.es/red/article/ view/232351/179271

Crovi, D. (2002). Sociedad de la información y el conocimiento. Entre el optimismo y la desesperanza. Revista Mexicana de Ciencias políticas y sociales, 45(185), 13-33.

Del Prado, A. y Doria, M. (2015). Construcción de materiales didácticos en ambientes virtuales de aprendizaje. In II Simposio Argentino sobre Tecnología y Sociedad (STS)-JAllO 44 (Rosario).

Delgado, M. y Solano, A. (2009). Estrategias didácticas creativas en entornos virtuales para el aprendizaje. Actualidades Investigativas en Educación, 9(2), 1-21.

Donolo, D., Chiecher, A. y Rinaudo, M. (2004). Estudiantes, estrategias y contextos de aprendizaje presenciales y virtuales. In Primer Congreso virtual latinoamericano de educación a distancia.

Dueñas, F. (2017). Investigación y educación virtual: alternativas que favorecen la inclusión social. Inclusión \& Desarrollo, 4(2), 1.

Elizondo, A., Rodríguez, J. y Rodríguez, I. (2018). La importancia de la emoción en el aprendizaje: propuestas para mejorar la motivación de los estudiantes. Cuaderno de pedagogía universitaria, 15(29), 3-11.

Espinosa, E. (2016). La reflexión y la mediación didáctica como parte fundamental en la enseñanza de las ciencias: un caso particular en los procesos de la formación docente. Tecné, Episteme y Didaxis: TED, (40), 175-209.

Fainholc, B. (2006). Optimizando las Posibilidades de las TICs en Educación. Edutec. Revista Electrónica de Tecnología Educativa, (22), a070-a070. https://edutec.es/revista/index.php/edutec-e/article/view/502/236

Fasce, E. (2007). Aprendizaje profundo y superficial. Rev Educ Cienc Salud, 4(1), 2. 
Flores, A. y García, A. (2017). Sistema de aprendizaje ubicuo en ambientes virtuales/Ubiquitous Learning System in Virtual Environments. Revista Cubana de Educación Superior, 36(2), 27-40.

Forero, I. (2009). La sociedad del conocimiento. Revista Científica" General José María Córdova", 5(7), 40-44.

García, J. (2011). Modelo educativo basado en competencias: importancia y necesidad. Actualidades investigativas en educación, 11(3).

García, L. (2012). La pedagogía problemática: fomento de una cultura del pensamiento crítico. REDHECS: Revista electrónica de Humanidades, Educación y Comunicación Social, 7(13), 6-19.

García-Peñalvo, F.y Pardo, A. (2015). Una revisión actualizada del concepto de eLearning. Décimo Aniversario. Education in the Knowledge Society, 16(1), 119-144. https://www.redalyc.org/pdf/5355/535554757008.pdf

Hernández, R. (2017). Impacto de las TIC en la educación: Retos y Perspectivas. Propósitos y representaciones, 5(1), 325-347. Hidalgo, S., Orozco, M.y Daza, M. (2015) Trabajando con Aprendizaje Ubicuo en los alumnos que cursan la materia de Tecnologías de la Información. Revista Iberoamericana para la Investigación y el Desarrollo Educativo: RIDE, 2015, vol. 6, no 11, p. 605-621. José, F. (2016). La comunicación. Salus, 20(3), 5-6.

Londoño, J. (2011). La investigación formativa en entornos virtuales. Revista Virtual Universidad Católica del Norte, 1(34), 1-7. https://revistavirtual.ucn.edu.co/index.php/RevistaUCN/article/view/327/624

Lozano, S. (2014). Prácticas innovadoras de enseñanza con mediación TIC que generan ambientes creativos de aprendizaje. Revista Virtual Universidad Católica del Norte, 4(43), 147-160.

Luque, M. (2009). Dinámica del aprendizaje y de la mediación en aulas virtuales: una visión desde la perspectiva de la formación humana. Organización de los Estados Americanos, Departamento de Asuntos Educativos.

Martí, J. (2009). Aprendizaje mezclado (B-learning) Modalidad de formación de profesionales. Revista Universidad EAFIT, 45(154), 70-77.

Martin, M. (2007). Evolución e historia en el desarrollo de la comunicación humana. M. Serrano, Teoría de la comunicación. La comunicación, la vida y la sociedad, 161-164.

Mas-Torelló,Ó.y Olmos-Rueda,P.(2016).El profesoruniversitarioenelEspacioEuropeodeEducaciónSuperior:laautopercepción de sus competencias docentes actuales y orientaciones para su formación pedagógica. Revista mexicana de investigación educativa, 21(69), 437-470. http://www.scielo.org.mx/scielo.php?pid=S1405-66662016000200437\&script=sci_arttext Muñoz, H. (2016). Mediaciones tecnológicas: nuevos escenarios de la práctica pedagógica. Praxis \& Saber, 7(13), $199-221$.

Oliva, P., Narváez, C. y Buhring, K. (2016). Valoración del mlearning en el proceso de aprendizaje de estudiantes de la Salud. Educación Médica Superior, 30(4), 372-381. http://scielo.sld.cu/scielo.php?pid=S0864-21412016000400013\&script=sci_ arttext\&tlng=en

Olivar, A. y Daza, A. (2007). Las tecnologías de la información y comunicación (TIC) y su impacto en la educación del siglo XXI. Negotium, 3(7), 21-46.

Pina, F., Kurtz, R., Brantes, J., Freitas, A., Da Silva, J. y Junqueira, C. (2016). Adoção de m-learning no ensino superior: o ponto de vista dos professores. REAd-Revista Eletrônica de Administração, 22(2), 279-306. http://bit.ly/2rca4×3

Pérez, L. (2014). Tecnología e informática de la historia. Resistencias e innovaciones en el uso investigativo y pedagógico de las TICs. Orbis. Revista Científica Ciencias Humanas, 10(29), 67-93. https://www.redalyc.org/pdf/709/70932556004.pdf

Pérez, M. y Telleria, M. (2012). Las TIC en la educación: nuevos ambientes de aprendizaje para la interacción educativa. Revista de Teoría y Didáctica de las Ciencias Sociales, (18), 83-112.

Ramírez, D. y Chávez, L. (2012). El concepto de mediación en la comunidad del conocimiento. Sinéctica, (39), 01-16.

Rivera-Vargas, P., Alonso-Cano, C. y Sancho-Gil, J. (2017). Desde la educación a distancia al e-Learning: emergencia, evolución y consolidación. Revista educación y tecnología, (10), 1-13.

Rodríguez-Hoyos, C. y Álvarez, M. J. Á. (2013). Análisis didáctico de las aulas virtuales. Una investigación en un contexto de educación superior. Edutec. Revista Electrónica de Tecnología Educativa, (44), a239-a239.

Rodríguez, M. y Barragán, H. (2017). Entornos virtuales de aprendizaje como apoyo a la enseñanza presencial para potenciar el proceso educativo. Killkana sociales: Revista de Investigación Científica, 1(2), 7-14.

Rojas, C.y Aguirre, S. (2015). La formación investigativa en la educación superior en América Latina y el Caribe: una aproximación a su estado del arte. Revista Eleuthera, 12.

Ruiz, C. y Antonio, A. (2016). Propuesta de buenas prácticas de educación virtual en el contexto universitario. Revista de Educación a Distancia, (49).

Salinas, J. (2012). La investigación ante los desafíos de los escenarios de aprendizaje futuros. RED. Revista de Educación a Distancia, 32. https://revistas.um.es/red/article/view/233091

Salinas, J.,DeBenito,B.,Pérez,A.y Gisbert,M.(2018).Blendedlearning,másalládelaclasepresencial.RIED.Revistalberoamericana de Educación a Distancia, 21(1), 195-213. https://www.redalyc.org/jatsRepo/3314/331455825011/331455825011.pdf Sierra, C. (2012). Educación virtual, aprendizaje autónomo y construcción de conocimiento: Libro de resultados de investigación. Institución Universitaria Politécnico Grancolombiano, 2012. 
Silva, J. (2010). El rol del tutor en los entornos virtuales de aprendizaje. Innovación educativa, 10(52), 13-23.

Tunnermann, C. (2011). El constructivismo y el aprendizaje de los estudiantes. Universidades, (48), 21-32.

UNESCO (2008) Estándares de Competencia en TIC para Docentes. Organización de las Naciones Unidas para la Educación, la Ciencia y la Cultura (UNESCO). http://eduteka.icesi.edu.co/pdfdir/UNESCOEstandaresDocentes.pdf

Villarreal-Treviño, M. (2006). La importancia de las estrategias de enseñanza en el logro del aprendizaje en alumnos universitarios. Trabajo de obtención de grado, Maestría en Educación y Procesos Cognoscitivos. Tlaquepaque, Jalisco: ITESO. Yahya, S., Ahmad, E. y Abb, K. (2010). The definition and characteristics of ubiquitous learning: A discussion. International Journal of Education and Development using Information and Communication Technology (IJEDICT), 6(1), 117 - 127. 